\title{
Usability Evaluation of sMouse: Software-Interface that Provides Sensory Reality of Use
}

\author{
Yui Tanaka *, Takayuki Fujimoto *
}

\begin{abstract}
Because of smartfication in societies, various kinds of physical interfaces have become softwarized. Regarding mobile environments, devices are designed on the basis that users do not need to use physical keyboards or mice. Most of the softwareized interfaces have higher performance than physical ones. However, there are many people who feel that the softwarized interfaces are hard to use despite of their high performance. They carry physical keyboards or external mice instead of softwareized interfaces. We suppose that this phenomenon is caused because humans want "tangible feeling of operations (the use)" when they use computer or machine interfaces. Almost all software-interfaces are hard to provide humans with "tangible feeling of operations (the use)". Implementing "sensory reality of the use" to the applications integrated to the mobile devices is indispensable to the software-interfaces. We proposed "sMosue", which is the application (app) that turns a smartphone into a mouse. With sMouse, the users can obtain the mice interfaces utilizing smartphones, which most people always bring .with them at all times. The user can use a smartphone as a mouse without carrying a physical mouse, streamlining of works is expected. In this paper, we describe outline of sMouse and its development purpose. In addition, we also explain the newly added function to provide "tangible feeling of operations (the use)", and performed its usability evaluation.

Keywords: Computer Mouse, IT, Human Interface, Internet of Things, Smartphone Application
\end{abstract}

\section{Introduction}

A computer mouse is an indispensable device to use a computer. Mouse has a longer history than expected. Since it was implemented by Douglas Engelbart (1925-2013) in 1968, it has continued to evolve up to the present. In 1991, Logitech Inc. developed a wireless mouse. Then, accompanied with improvement of wireless technology and battery function, and the evolution for high accuracy and miniaturization are still in progress to meet a high demand. In recent years, pursuit of higher quality and miniaturization of laptop computers and the increasing use of cloud services are also prominent. They generalized, the environment so-called "mobile office". It can be said that a wireless mouse is suitable for the lifestyle in which we can work anywhere if there is an Internet connection.

\footnotetext{
* Toyo University, Tokyo, Japan
} 
Usually, a laptop computer has a track pad instead of a mouse. Its quality has been also improved spectacularly. In addition, other than a track pad, there are many alternatives as input interfaces. Today, a mouse is no longer an indispensable item to carry with a laptop computer. For example, today, there is a high demand for touchscreen laptops with which the users can directly tap the screen to operate the computer. It reflects the social situation that smartphones and tablets are popular. There are systems that the users can operate by eye movements. They are mainly for physically challenged people, and those systems undergo intense studies with high interests. If the development of the systems is completed, they will be very useful tools that can be operated only by wearable devices such as glasses. Also, Tsuzaki tried to operate a computer by gestures [2]. The users can operate the proposed system only by small devices to put on the fingers. Space-conserving is expected and the users do not have to carry normal mice. Sakata is developing a system upon which the users can operate the projected images by feet [3]. Even though both hands are not free, the users can operate the system and also zoom the image to be displayed. Kikuchi tried to develop a system that can be operated by earphones and they uti-lized ears as interfaces. There are a limited number of the input commands that can be manipulated, however, simple operations become easier regardless of locations.

Thus many kinds of input devices have been devised and commercialized. However, there are many people who like mice that we have been familiar with for a long time, and they still use mice as interfaces. In fact, a mouse continues evolving, and mouse grip designs, pointing accuracy and customizability has been improved. In this way, a mouse is an optimized device for the uses of computers.

However, today, the small laptops have various functions. In addition, every data is stored in the cloud service. Those technologies have generalized the mobile office environment. The number of the people who avoid carrying external devices is increasing. Although there is a great need for mice, to carry the external devices does not suit to modern lifestyles. Thereupon, we proposed the app called "sMouse" to turn the smartphone to an external mouse. The app provides the equivalent operability with the mouse without carrying the external device.

\section{Purpose and Aim}

The concept of sMouse is "sensory reality" for its use. The detail will be described using a term, "analog operability" upon "3.1 Concept". In recent years, various tools like calculators, books, and calendars have digitized as applications and also laser projection keyboards have appeared. As the society changes to so-called "Smart society", the physical interfaces have gone. The digitized interfaces have higher specs than the physical interfaces, however, they still remain as commodities in reality.

Why is there a demand for the analogical tools now? In operability between analog objects, which exists in reality, and applications, there is a big difference: with or without "sensory reality". The operations for applications on mobile devices only cause sensations of tapping or sliding a flat surface. There are no operations like pushing buttons, turning pages, or moving the objects. However, the actual objects require the users to perform more operations that cause sensory reality. Regarding the operations for application, the users cannot acquire tangible feeling. Because applications do not provide tangible feeling, the users tend to do wrong operations or repeat same operations. That is why the users have an impression that digitized inter- 
faces are useful, however they are hard to use. On the other hand, it is clear that applications bring us not only high specs but also space-saving and efficiency of works.

What we need to research is how to implement "sensory reality of the use" on the proposed application for the society in which various objects are integrated to a smartphone. By adding "sensory reality of use" effect to applications integrated in the mobile devices, we suppose that the applications will become easier to use and it leads to enrich the smart society. In this study, we aim to improve operability by implementing a function to provide sensory reality (of the use) to sMouse. In addition, we conduct an evaluation experiment of the influence on operability under the condition that functions to provide sensory reality (of the use) are implemented to the software interface, and we verified its effectiveness.

\section{About "sMouse"}

"sMouse" is an application that we have developed [1]. The users can use sMouse as an extra device without carrying an external device because the implemented smartphone app works as a substitute for the external device.

\subsection{Concepts}

sMouse has mainly two concepts. One is "analog-like operability", which is associated with sensory reality for the use of the interface, and the other is "prevention of being from copied" by implementation of "analog-like operability" regarding the design.

\subsubsection{Analog-like Operability}

Analog-like operability of sMouse is based on the idea to incorporate "analog" into digital. Today, because of IT development, recently electric appliances and other utensils are controlled by computers behind our sight and they acquire data and show the optimum function for users or consumers. The idea of IoT (Internet of Things) is also common, and every kind of device or environment is connected to the Internet for digitization.

Our aim is not the practice of IoT, with which various objects are connected to the Internet and digitalized. In contrast, We aim to practice the idea to add an analog-like tool or operability to the digital devices or environments because we suppose that it leads to the realization of the truly user-friendly devices. The term "analog-like operability" means an operational quality with which the user can intuitively acquire tangible feeling of operating the applied devices. Operation system of sMouse is different from the other previous mouse apps. The system requires the user to hold, grip, and move the smartphone itself. This enables the user to get tangible feeling like using a physical mouse. In this paper, we refer to this kind of operability, which provides sensory reality as "analog-like operability".

\subsubsection{Prevention of being from Copied}

We also suppose that "analog-like operability" can prevent the app design from "pakuri". In recent years, "pakuri (in this paper, it is defined as "taking" that cannot be said to be a crime in most of the context, but is the act which is not supposed to do by someone else in Japanese. It can sometimes mean a crime depending on the context)" problems including right or wrong discus- 
sion are occurring frequently regarding digital tools and environments. That is because most of the similarities originates from internal program, and those similarities are difficult to notice intuitively. Regarding the Internet, various services or software are appearing. When one service gains popularity or starts spreading, many similar services and software appear. It is common that the service released later solves the problems of the preceding service, and acquires the reputation as a better service.

Of course, patents protect the services or software, however the similarities often develop to be a legal conflict including accusations. In many cases, recent new services or software on the Internet start from small ideas of individuals, and they rapidly develop to be a scale that cannot be managed at the individual level. It is not always the case that the users apply for or acquire patents beforehand. It is difficult to keep away the new ideas of mechanisms or systems from becoming victims of "pakuri". On the other hand, the uniqueness of visual designs or interfaces, and the gimmicks or systems that users can experience with their physical senses like convenient goods, increase the possibility to prevent themselves from being copied. Because everyone can see the obvious similarity in appearance intuitively, there are few people who seek to copy the visual design. "Analog-like operability" enable the users to acquire tangible feeling of operation and it is easy to find out if there is similar operability. Because pakuri will soon be discovered, there are few people who positively copy the interface with "analog-like operability". sMouse is effective not only for space-saving of mobile office, but also for one of the attempts based on the concept: "the design that prevents being from copied".

\subsection{Existing Other Mouse Apps}

All mouse apps released currently are remote mouse apps. The user can operate a pointer on the computer screen by tapping the touch screen of smartphone. These operations are similar to the ones for a remote track pad, and they are far from the actual operations for a physical mouse. Of course, it is difficult for the previous mouse apps to create sensory reality like using a physical mouse.

\subsection{Design of "sMouse"}

We presuppose the use on iPhone by Apple Inc. By acquiring position information using the accelerometer, which is equipped on iPhones. The smartphones can function as mice. In this section, we describe the execution image of sMouse in detail. The prototype, which was proposed in this paper, was designed in the motif of a mouse device. If the user taps on the clicking part like a normal mouse, the user can perform click operation on the computer connected by Bluetooth. How to use is also same as a mouse. The user can control the cursor on the computer by moving the smartphone back and forth and around like a physical mouse. The app does not have any other features like functions to operate PowerPoint or music player. It has a mouse function only. This is because mice we usually use do not have such functions.

Bluetooth is used for the connection to the computers. There are two reasons. One is to prevent operations by other people who use the same app, as the situation is seen with the existing apps. The other is to enable the operation for the computers in environments without Wi-Fi. Today, it has become common for people to carry computers. However, it is not always the case 
that where one is going has Wi-Fi environment. Some cafés are supplying services for Wi-Fi environments. However, most of the case, the services require charges. The use of sMouse presupposes that the distance between the computer and the mouse is close. Therefore, Bluetooth connection is better than Wi-Fi connection because of low price and functionality that works in any environment.

Fig. 1 shows the transition diagram of sMouse. By tapping $<$ START $>$ button on Main screen, the screen switches to the next screen. In this stage, the smartphone is not connected to the computer yet. When the user taps $<$ CONNECT $>$ button, the screen switches to Connected screen and, the smartphone will be connected to the computer that has recognized the smartphone in advance. In this mode, the user can use the smartphone as a mouse device. If the user taps $<$ DISCONNECT $>$ button at the bottom of the screen, the connection is deactivated, and the screen will switch back to the previous screen. Fig. 2 shows execution image of the prototype app.

\section{Main screen}

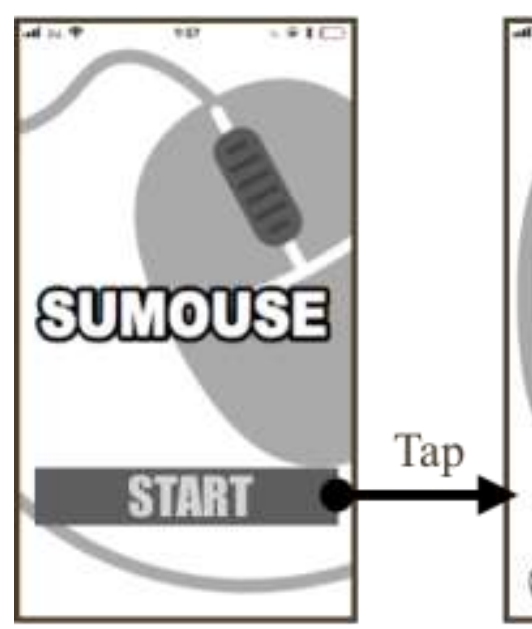

Figure 1: Transition diagram of the app
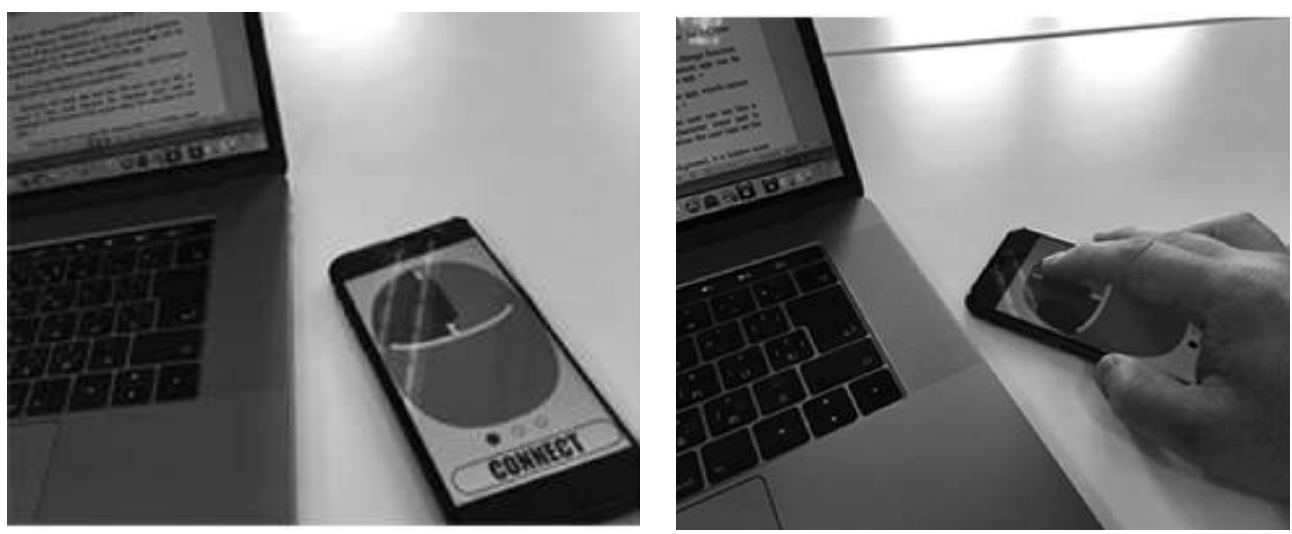

Figure 2: Prototype sMouse 


\subsection{Design of "sMouse"}

sMouse that is accompanied with actual moves like "holding", "dragging", and "pushing" is closer to a physical mouse than software interfaces displayed on screens or track pads with which the users cannot gain tangible feeling of the use. We added a function that provides users with "sensory reality of operation", and performed its usability evaluation.

\section{Evaluation of sMouse Operability}

In this study, we focused on "sound and vibration" as elements to provide sensory reality of operating a mouse. Almost all physical mice in the market, have click buttons that slightly sink when users click them. It gives the users tangible feeling of pushing the button. In accordance with that, the mouse makes "clicking" sound. The scroll wheel provides tangible feeling of rotation and "rotating" sound when the wheel is rotated. Those delicate sounds and vibrations give users comfort and "tangible feeling of using devices" We suppose that they influence on work efficiency for the tasks to be processed with computers. Thereupon, we added a function that replicate "sensory reality of operation" to sMouse using sounds and vibrations. We investigated how sensory reality of "sounds and vibrations" effects on operability of the interface.

\subsection{Evaluation Method}

We created four kinds of sMouse apps that different actions are setup for mouse-button-clicking. We conducted a questionnaire survey on 22 males and females in their teens and 20s. Table1 shows the click action details. On the screen for operations, the same mouse pictures are to be displayed.

Table.1: Application1 4 Click Action Event

\begin{tabular}{llccl}
\hline App & App name & Sound & Vibration & \\
\hline \hline App1 & Plain App & $\times$ & $\times$ & None \\
App2 & Sound App & $\bigcirc$ & $\times$ & Clicking sound \\
App3 & Vibration App & $\times$ & $\bigcirc$ & Vibrations \\
App4 & Mixture App & $\bigcirc$ & $\bigcirc$ & Clicking sound and Vibrations \\
\hline
\end{tabular}

The examinees used those apps 1 to 4 in turn, compared each other, and evaluated their user-friendliness. 


\subsection{Result of Evaluation}

Table 2 shows result of evaluation. Numbers are rounded down to the second decimal place.

Table.2: Result of Evaluation (Unit: \%)

\begin{tabular}{lccrr}
\hline & User-friendly & No difference & User-hostile & Don't know \\
\hline \hline App 2 & & & & \\
\hline Compared with App1 & 81.8 & 13.6 & & 4.5 \\
\hline App3 & & & & \\
\hline Compared with App1 & 77.2 & 9.0 & 9.0 & 4.5 \\
\hline Compared with App2 & 45.4 & 22.7 & 31.8 & 0 \\
\hline App4 & & & & \\
\hline Compared with App1 & 81.8 & 0 & 13.6 & 9.0 \\
\hline Compared with App2 & 45.4 & 13.6 & 36.3 & 4.5 \\
\hline Compared with App3 & 50.0 & 22.7 & 22.7 & 0 \\
\hline
\end{tabular}

\subsection{Result of Evaluation}

We consider the comparison result of each application.

\subsubsection{App 1 (Plain App)}

App 1 is the most difficult to use, compared with any of 2, 3 and 4. From this, it turned out that implementing some action for clicks gives the user-friendly impression.

\subsubsection{App2 (Sound App) and App3 (Vibration App)}

Comparing sound with vibrations, there are more people who feel that app is slightly easier to use with vibrations rather than clicking sound. As a reason, it can be inferred that the users are stimulated more with vibration rather than sound alone as "sensation". There is a feeling of the click button's bouncing back when pressing the button of a mouse. Vibrations are different from that, however, because similar feeling can be obtained, it seems that there are many people who find that the application with vibrations is user-friendly.

However, when comparing App 1 to App 2, there is no one who finds that App 2 is harder to use, whereas some people evaluate App3 as " User-hostile " when comparing App 1 with App 3. It means that vibration effect is not necessarily easy to use.

When operating a smartphone as a mouse, since it is placed on the desk for operations, the vibration sounds can be noisy because of the echo. That was pointed out at the time of the evaluation experiment. A normal mouse make "clicking" sound. However, the vibration sound is 
dull sound like "buzzzz" which is unfamiliar with the users when operating the normal mouse. It seems that some people feel a sense of incompatibility.

\subsubsection{App4 (Mixture App)}

Compared to App 1, it seems that there are many people who feel that App 4 is easy to use as with App 2 and 3. However, when comparing App4 with App 2 and App 3, the results vary. Overall, the percentage of the people who feel that App 4 is easy to use is the largest, however comparing the usability of App 4 with App 2, the difference in the percentage is only 2 points. Since there is no big difference, the usability of App 2 also cannot be ignored.

The cause of the dispersion of the result is that the vibration, "buzzzz" was not made at the timing of "clicking" sound. Ideally, vibrations that match to "clicking" sound are to be made. It is necessary to adjust the length and strength of the vibration to make them as close as possible to the impression of the clicking sounds.

Taking as a whole of the evaluation results, there are few people who selected "No difference" or "Don't know", and the percentage is less than 5 points. Therefore, it is clear that setting the action events at the time of clicking affects operability. We found that operability is improved when "sensory reality of clicking" is implemented. Vibrations are most effective when implementing "sensory reality of clicking" and the sound is second-best. However, if these are both made at the same timing, the evaluation on operability becomes unstable. We need to be careful. It is necessary to set them in the way that matches both as stimulation.

\section{Conclusion}

In this paper, we evaluated usability of sMouse, which turns a smartphone into a mouse. We implemented "sensory reality of operation", which is the concept of sMouse by sound and vibrations, and conducted questionnaire survey. As a result, we found that app with "sensory reality" is more user-friendly than plain app. In addition, it became clear that vibrations are slightly more effect ive than sound. This fact can be also applied to the other apps, and it can be expected to be the important guideline when considering future operability of smartphone apps.

Since it was found that we need to match sound and vibrations as stimulation, it is necessary to consider how to set them concretely. It is also required to verify difference in operability comparing the combination of sound and vibration effects with the cases in which sound or vibration effect is set alone.

In this paper, we conducted evaluation survey only for the click function, however a mouse also has a scroll wheel function. Regarding this function as well, we will produce sensory reality of operation and perform an evaluation survey to improve usability.

\section{References}

[1] Yui Tanaka, Takayuki Fujimoto. "A Design of Application to Turn a Smartphone into a Computer Mouse and Possibility of Preventing from Being Copied", 7th International Congress of Advanced Applied Informatics, 2018.7.11. 
[2] Takashi Tsuzaki, Koji Hashimoto, Tomu Tominaga, Yosjinori Hijikata. "Implementation of Computer Mouse Functions by Gestures"[in Japanese], Fukuoka University review of technological sciences 89, pp43-48, 2012-09.

[3] Nobuchika Sakata, Fumihiro Sato, Tomu Tominaga, Yoshinori Hijikata. "User Study of Floor Interaction with Wearable Projection Interface Using Hand and Toe" [in Japanese], Transactions of the Institute of Systems, Control and Information Engineers, vol.31, no.3, 112-120, 2018.

[4] Takashi Kikuchi, Katsutoshi Masai, Maki Sugimoto, Bruce H. Thomas, Yuta Sugiura. "EarTouch: Turning the Ear into an Input Surface" [in Japanese], The Virtual Reality Society of Japan, vol.22, no.4, 477-483, 2017.

[5] "Doug Engelbart's mouse prototype", The Computer History Museum", USA, http://www.computerhistory.org/revolution/input-output/14/intro/1876.

[6] Takayuki Fujimoto. "The problem of content design in the Internet age: How do theft and plagiarism happen?". 7th International Conference on Innovative practices in Business, Social Sciences and Humanities research, Dubai, UAE, 2017.

[7] Takayuki Fujimoto. "Therefore the designer is flaming" [in Japanese], Chuokoron-shinsha Inc, 2016.

[8] Takayuki Fujimoto. "A design that makes information easy to understand: What is information design and what is not it?" [in Japanese]. The journal of information science and technology association, vol.65, No11, Information science and technology association, japan, 2015.11, pp.450-456.

[9] K. Mine, M.Ibato, H. Hayashi, R. Otaki. "Evaluation of the input device DigiTrack for mobile information equipment [in Japanese]. Proceeding of the 64th National Convention of IPSJ, 2002, vol.64, no.4, p. 475-481.

[11] A.Saito, K. Nishida, Y. Tsujino, N. Tokura. Improvement of mouse driver for improved pointing accuracy [in Japanese], The Special Interest Group Technical Reports of IPSJ human computer interaction, 1998, vol.1998, no. 35, p. 51-56.

[12] N. Ozaki, S. Honda, T. Tanaka, K. Akita, Y. Sagawa. A Character Input Method for a Rectangular Smartwatch by Combination of Stroke Gesture and Tap[in Japanese], Transactions of Information Processing Society of Japan, 59(8), pp1511-1519, 2018-08-15.

[13] T. Yoshida, H. Igarashi. Development of an input interface for operation mastery support by vibration stimulation[in Japanese], Conference on Information, Intelligence and Precision Equipment: IIP 2018, Vol.0, pp02-1, 2018.

[15] K. Ikematsu, I. Siio. Trackpad-based Mode Switching Technique Using Carbon Copy Metaphor, Transactions of Information Processing Society of Japan 58(5), 1014-1024, 2017-05-15.

[16] S. Sirakami, Y. Kinoshita. User Impression Evoked by Various Smartphone Vibration Patterns, The transactions of Human Interface Society 18(1-4), 403-414, 2016. 
[17] M. Tsuchiya, L. Tanaka. Design of the interface for interactive magazine contents by using a smart-phone, Japanese Society for the Science of Design, Journal of the Science of Design, 63(0), 20, 2016.

[16] H. Takahashi, M. Ban. Ambiguous user interface that drives our imagination, The Special Interest Group Technical Reports of IPSJ. Human Computer Interaction(HCI), 2015-HCI-163(9), 1-4, 2015-05-07.

[17] Yui Tanaka, Takayuki Fujimoto, "Proposal for a Font Changer Application Based on Results of an Impression Evaluation Experiment", International Journal of Computer Science \& Network Security,Vol.18 No.6, 2018

[18] Yui Tanaka, Takayuki Fujimoto, "Proposal of Physical Control Interface for smartphones to Improve Readability" ,3rd International Conference on Control Engineering and Artificial Intelligence(CCEAI 2019), 2019

[19] Yui Tanaka, Takayuki Fujimoto, "Proposal for 9-Squares Shogi Application Utilizing 3D Hologram", The 2nd International Conference on Applied Cognitive Computing (ACC'18), 2018

[20] Risa Ogawa, Takayuki Fujimoto, "The possibility of 3D-origmi system as a tourism promotion tool for Japan", Journal of Advanced Research in Social Sciences and Humanities Volume 3, Issue 1, 2018

[21] Risa Ogawa, Takayuki Fujimoto, "The possibility of 3D-ORIGMI system as a tourism-promotion tool for Japan", 7th International Conference on Innovative practices in Business, Social Sciences and Humanities research, Dubai, UAE, 2017

[22] Risa Ogawa, Takayuki Fujimoto, "The development and evaluation for Japanese "Origami" application with 3D expression", 25th International Conference on Systems Engineering, USA, 2017

[23] Ziran Fan, Takayuki Fujimoto, "Proposal of a Design Method to Apply the Analog Features to Digital Media", 3rd International Conference on Control Engineering and Artificial Intelligence(CCEAI 2019), 2019

[23] Ziran Fan, Takayuki Fujimoto, "Proposal of a Scheduling App Utilizing Time-Perception-Reality in Analog Clocks", 1st International Conference on Interaction Design and Digital Creation / Computing (IDDC 2018), Japan, 2018

[23] Takayuki Fujimoto, "Ideology of AoD: Analog On Digital-Operating Digitized Objects and Experiences with Analog-like Approach", 1st International Conference on Interaction Design and Digital Creation / Computing (IDDC 2018), Japan, 2018

[24] Kazuya Murata, Eigo Ito, Takayuki Fujimoto, "A Proposal for "Infinite Scale" Ruler Application that Provides Analog-like "Sensory Reality"', 1st International Conference on Interaction Design and Digital Creation / Computing (IDDC 2018), Japan, 2018 